\title{
Zur Aktualität Richard von Weizsäckers Parteienkritik
}

Die demokratiepolitische Lektion, die Richard von Weizsäcker dem politischen Personal der Bundesrepublik in seinem ZEIT-Gespräch (1992) erteilte, löste eine kontroverse Debatte aus. Wenige Jahre nach der Friedlichen Revolution in der DDR und der deutschen Wiedervereinigung beklagte das deutsche Staatsoberhaupt einen beunruhigenden Ansehensverlust der Parlamente, ja der Politik insgesamt, und warnte vor Auswüchsen der Parteienherrschaft. Diese Kritik war alles andere als wohlfeil. Sie verriet die Sorge des Bundespräsidenten um die Zukunft und Funktionsfähigkeit unserer Demokratie. Zwanzig Jahre sind seitdem vergangen, doch von Weizsäckers Mahnungen haben sich nicht erledigt.

1. Unsere Demokratie wird ,angefressen“, infrage gestellt von ganz verschiedenen Seiten und durch sehr verschiedene Entwicklungen. Dazu zählen die Unzufriedenheit, die Ungeduld, der Verdruss vieler Bürgerinnen und Bürger. Dazu zählt das individuelle Fehlverhalten von Politikern, die die politische Klasse und die Institutionen der Demokratie immer mal wieder in einem trüben Licht erscheinen lassen. Dazu zählen aber auch - ganz vorsichtig ausgedrückt - leichtfertige Medien, die das Verständnis für und das Vertrauen in die Mühseligkeit demokratischer Prozesse und Entscheidungen nicht gerade befördern. Auf diese und viele weitere Phänomene hatte Richard von Weizsäcker 1992 hingewiesen. Sie belegen den Vertrauensverlust der repräsentativen Demokratie.

Aus meiner Wahrnehmung ist eine tiefer liegende Entwicklung wesentlich: Unsere Demokratie wird vor allem bedrängt durch die Dominanz ökonomischer Prozesse und Entscheidungen, die den Primat demokratischer Politik in Frage stellen. Das scheint mir der eigentlich alarmierende Vorgang. Man bedenke das Tempo und die Reichweite ökonomischer Prozesse einerseits und andererseits die Langsamkeit und Begrenztheit demokratischer, politischer Prozesse und Entscheidungen. Die Wahrnehmung der Diskrepanz zwischen beiden erzeugt - befördert durch eilige Medien - Ungeduld und Unzufriedenheit mit diesem demokratischen Prozess.

Parteien und Parlamente erwecken immer mehr den Eindruck, nur noch atemlos hinterherhetzen zu können, bestenfalls Wundpflaster und Schmerzmittel verabrei- 
chen zu können. Das Sagen, so der verbreitete Eindruck, haben die ominösen Märkte, haben Ratingagenturen, Finanzmanager und Banken. All das reicht fast bis zur Selbstabdankung demokratischer Politiker. Eine fatale Entwicklung! Denn Vertrauen in die Gestaltungsfähigkeit von Politik ist Voraussetzung für die Stabilität und Funktionsfähigkeit einer gesellschaftlichen und wirtschaftlichen Ordnung, von der nicht zuletzt auch alle Unternehmen der Finanz- wie Realwirtschaft abhängig sind. Sie sitzen mit auf dem Ast, an dem ein entgrenzter, enthemmter Finanzkapitalismus und seine marktradikalen Anhänger sägen. Die Schuldenkrise bedroht längst ganze Gesellschaften, stürzt Hunderttausende in Armut und Verzweiflung und spült zugleich neue Vorurteile, Misstrauen und Politikverachtung an die Oberfläche. Die Demonstrationen in Griechenland, Portugal und anderen Ländern gehen mit bestürzenden Wutausbrüchen einher, die den gesellschaftlichen Frieden gefährden. Demokratische Politik muss die Gestaltungshoheit über die Finanzwirtschaft und den wirtschaftlichen Wettbewerb zurückgewinnen. Sie muss neue Leitplanken zur Regulierung der Finanzmärkte errichten, deren Erpressungspotenzial verringern, systemische Risiken frühzeitig bekämpfen und die grenzüberschreitend tätigen Banken effektiv kontrollieren. Wir brauchen eine andere Bankenkultur. $\mathrm{Zu}$ dieser gehört dann auch die Umsetzung einer ganz selbstverständlichen Einsicht der Einsicht, dass die Finanzmärkte an den Kosten der bisherigen Rettungsmaßnahmen zu beteiligen sind, zum Beispiel durch eine Finanztransaktionssteuer.

2. Richard von Weizsäcker hat mit seiner Parteienkritik eine fruchtbare Debatte angestoßen, an der sich bis heute Politiker, Politikwissenschaftler, Journalisten, Bürgerinnen und Bürger beteiligen. Allerdings hat sich, so meine Wahrnehmung, der Ton der Debatte geändert, er ist schärfer geworden, polemischer, grundsätzlicher. Mitunter nimmt die Kritik an den demokratischen Parteien demokratiegefährdende Züge an. Berechtigte Kritik mündet immer häufiger in einen medialen Überbietungswettbewerb, der Grenzen des Anstands und der Fairness sprengt. Lustvoll wird eine Negativspirale bedient, die den kritisierten Akteuren kaum noch Handlungsspielraum lässt. Doch mediale Übertreibungen, Häme, Zynismus tragen nicht zur Überwindung von Unsicherheit und Misstrauen bei, im Gegenteil. Parteienkritik unterliegt einem Wettbewerb, an dem sich auch politische Parteien selbst beteiligen.

Grundsätzlich ist Politik, sind die Parlamente vor allem Orte der Moderation, also der Mäßigung und des Ausgleichs von Partikularinteressen, von divergierenden Gerechtigkeitsvorstellungen und Bedürfnissen, von Gerechtigkeitskonflikten. Diese Aufgabe der Parlamente wird durch verschiedene Entwicklungen und Haltungen jedoch zunehmend in Frage gestellt. Eine davon ist die Überzeugung, dass Parlamente und Parteien als Orte der Demokratie diese Rolle nicht mehr ausreichend erfüllen und damit ihre Schlüsselstellung in der Demokratie zu verlieren beginnen, 
wie Protagonisten und Publikum der Piraten-Partei meinen. Ich sehe das nicht so! Die Parteien, erst recht Volksparteien, haben bzw. behalten wider allen Verdruss eine unersetzliche Aufgabe: Sie erarbeiten, sie bilden in ihren Reihen die Kompromisse vor, die Konsense zwischen den widerstreitenden Interessen und Meinungen, von denen die demokratische Politik, die Gesellschaft insgesamt leben. Denn Demokratie ist ja nicht nur Streit und Konflikt, sondern immer auch der Versuch, Kompromisse und Konsense zu bilden und zu Entscheidungen zu kommen. Betrachtet man allerdings die Stimmengewinne der Piraten-Partei in den letzten Landtagswahlen, so scheinen die strukturierenden und ausgleichenden und insofern immer auch mühseligen und grauen Verfahren in und zwischen den Parteien irgendwie eine antiquierte Form der demokratischen Befriedigung gesellschaftlicher Konflikte zu sein. Die Piraten selbst kokettieren damit, unfertig und unverbindlich zu sein. Stattdessen bieten sie über das Internet Mitmachmöglichkeiten, stilisieren sich als Anti-Partei und werben offensiv damit, keine festen Positionen und Überzeugungen zu haben.

Für langjährige Parlamentarier ist das etwas durchaus Irritierendes und Befremdliches, doch zugleich auch etwas Spannendes: Die Piraten machen ein Angebot für das Mitmach- und Beteiligungsbedürfnis von Menschen, die offensichtlich die etablierten Parteien nicht mehr erreichen - Liquid Democracy, zu Hause sitzen, anonym bleiben, aber sich trotzdem beteiligen an Meinungsbildungsprozessen. Das macht sie attraktiv und das wird für die Volksparteien eine Herausforderung sein. Die zur Schau gestellte Naivität und Unverbindlichkeit macht sie vielen sympathisch. Aber Piraten sollten auch wissen: Dauerpalaver, ohne den Mut zur Entscheidung, bleibt folgenlos.

Die Bürger haben einen Anspruch darauf, dass eine Partei eine erkennbare Position verbindlich vertritt und auch die Verantwortung für sie übernimmt. Sind die Piraten eine neue innovative Kraft, die sich stabilisiert, oder sind sie lediglich eine neue Projektionsfläche für die alte Politikdistanz? Diese Frage ist noch nicht gänzlich entschieden. Die Piraten spekulieren durchaus mit populistischen Vorbehalten und verstärken sie damit: „Politiker haben keine Vorstellung, wie normale Menschen leben", so ließ sich Martina Weisband, einstige politische Geschäftsführerin der Piraten-Partei, von Bild zitieren. Und im Berliner Wahlkampf war auf Plakaten der Partei zu lesen: „Wir sind die mit den Fragen, Ihr seid die mit den Antworten“. Die spannende Frage bleibt: Wie wird sich das auf Dauer vertragen: Liquid Democracy, ein Angebot von Mitwirkungsmöglichkeiten bequemer Art einerseits und anderseits Kenntlichkeit, Verbindlichkeit, Verantwortlichkeit, von denen Demokratie lebt? Transparenz und Anonymität - beides zusammen geht nicht. 
3. Auf die Frage nach der „Machtversessenheit“ und „Machtvergessenheit" der Parteien hatte Richard von Weizsäcker selbst die beste Antwort gegeben: „In einer Demokratie kommt es auf die Gesellschaft im Ganzen an, auf ihren Willen, ihre Moral, ihre Einsicht, ihren Geist, dagegen nicht allein auf Parteien." Richtig! Die Demokratie bliebe leer, abstrakt, formal, wenn sie nicht durch die Bürgerinnen und Bürger in Anspruch genommen und mit Leben erfüllt würde, durch Bürgerinnen und Bürger, die ihre legitimen Interessen und begründeten Meinungen vertreten und die über ihren verständlichen Egoismus hinaus auch Vorstellungen vom Gemeinwohl entwickeln. Wenn aber Bürger vielfach das Gefühl haben, dass sie dies nicht mehr oder nicht ausreichend im Rahmen des demokratischen Regelwerks und des vorhandenen Institutionengefüges tun können, dann nimmt ihr Engagement die Form des Protestes an. Bürger, die aus unterschiedlichsten Gründen enttäuscht sind von der Parteiendemokratie, deren Vertrauen in die repräsentative Demokratie geschwunden ist, gehen auf die Straße, geben ihren Zorn in Umfragen kund, wählen eine inhaltlich noch gänzlich unbestimmte Partei. Oder sie gehen gar nicht erst zur Wahl. Wahlverzicht ist ein besonders fataler Ausdruck des Misstrauens. Hier fehlt das Grundvertrauen in die eigenen Möglichkeiten, etwas verändern und etwas gestalten zu können. Dieses Misstrauen trifft nicht nur eine Partei, sondern unser Gemeinwesen insgesamt, es schädigt unsere Demokratie. Auf Dauer erweist es sich als Einfallstor für Populisten und Demagogen, für all jene, die einfache Antworten und schnelle Lösungen versprechen.

Die Parteien, wir Politiker sollten lernen, alle unsere Entscheidungen so transparent wie überhaupt nur möglich vorzubereiten und zu fällen, damit die interessierten Bürger erkennen können, ob und wann und wie sie sich rechtzeitig und folgenreich einmischen können, wenn sie es denn wollen. Die Parteien sind schließlich auch wichtiger Teil der Zivilgesellschaft und nicht einfach Teil des Staatsapparates!

Mehr Möglichkeiten zu direkter Bürgerbeteiligung nützen unserer Demokratie, davon bin ich überzeugt. Auch zwischen den Wahlen als Staatsbürger sich melden zu können und aktiv eigene Anliegen nicht bloß passiv und unverbindlich, wie bei Meinungsumfragen, vertreten zu können, das befruchtet die Arbeit der politischen Parteien, macht aber die Parteien wahrlich nicht überflüssig. Ich bin für die Instrumente der Volksgesetzgebung auch auf Bundesebene - also für Volksinitiative, Volksbegehren, Volksentscheid nach klar definierten Regeln. Diese Instrumente können Vertrauen stärken, sie ermöglichen, dass die Zivilgesellschaft vielleicht einen ähnlichen Einfluss auf Politik haben könnte, wie es gegenwärtig Lobbyisten haben. 
Korrespondenzanschrift:

Dr. h. c. Wolfgang Thierse, MdB

Vizepräsident des Deutschen Bundestages

Platz der Republik 1

11011 Berlin

E-Mail: wolfgang.thierse@bundestag.de 